\title{
Reparo ósseo induzido por diferentes substitutos de enxerto ósseo em defeitos de tamanho crítico na calvária de ratos.
}

\author{
Mauricio Andrés Tinajero ARONI (1), Paulo Firmino da COSTA NETO (1), \\ Guilherme José Pimentel Lopes de OLIVEIRA (2), Rosemary Adriana \\ Chiérici MARCANTONIO (1) e Elcio MARCANTONIO JUNIOR (1)
}

\section{ARTIGO ORIGINAL}

\section{Resumo}

Introdução: A utilização de substitutos ósseos em procedimentos de enxertia como alternativa ao uso de enxerto ósseo autógeno tem sido indicada, entretanto, a comparação direta entre esses biomateriais tem sido pouco explorada.

Objetivo: Avaliar o efeito de diferentes substitutos ósseos osteocondutores no reparo ósseo em defeitos de tamanho crítico (DAC) em calvária de ratos.

Material e método: Um DSC com diâmetro de $8 \mathrm{~mm}$ foi feito em cada um dos 40 ratos usados neste estudo. Os animais foram alocados aleatoriamente em 5 grupos $(n=8)$, de acordo com o tipo de substituto ósseo utilizado para o preenchimento do DSC: COA (Coágulo); AUT (osso autógeno); DBB (enxerto ósseo bovino desproteinizado); HA / TCP (cerâmica bifásica composta por hidroxiapatita e $\beta$ fosfato tricálcico); e TCP ( $\beta$-fosfato tricálcico). Uma análise microtomográfica foi realizada para avaliar o comprimento linear do defeito remanescente (DLL) do DSC e o volume dos tecidos mineralizados (TM) dentro do DSC aos 3, 7, 15 e 30 dias após o procedimento cirúrgico. Além disso, foi realizada uma análise histométrica para avaliar a composição do tecido ósseo reparado (\% Osso e\% Biomaterial) no período de 30 dias.

Resultado: Foi demonstrado que o COA apresentou o menor DLL e MT dentro do CSD. Além disso, o COA apresentou o maior\% de osso na DAG. O DBB apresentou maior MT e maior\% de partículas de substituto ósseo no CSD do que os grupos AUT e TCP. Os grupos DBB e AUT apresentaram maior\% de osso no DSC do que o grupo TCP.

Conclusão: $O$ uso do DBB promoveu melhor padrão de ganho e formação de volume ósseo em relação ao TCP e HA / TCP, mas foi biologicamente inferior ao AUT.

Palavras Chave: Histologia; microtomografia de raios-x; regeneração óssea; substitutos ósseos.

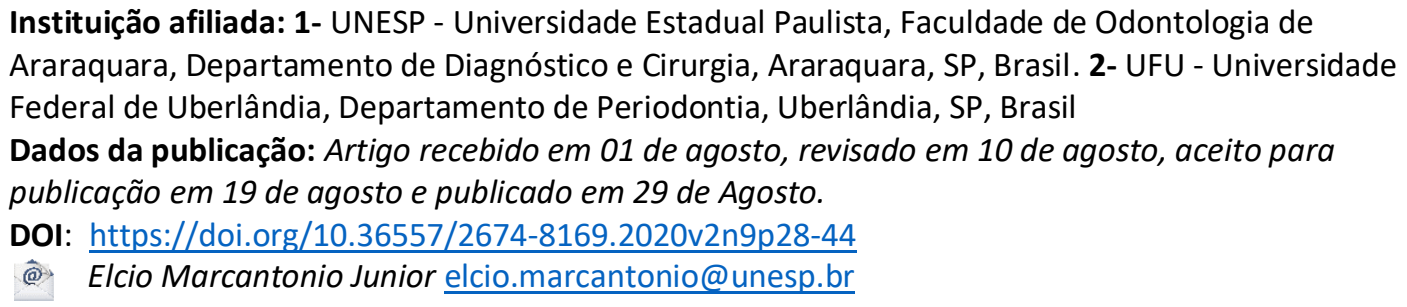




\section{Bone repair induced by different bone graft substitutes in critical- sized defects in rat calvaria.}

Introduction: The use of bone substitutes in grafting procedures as an alternative of the use of autogenous bone graft has been indicated, however, the direct comparison between these biomaterials has been little explored.

Objective: To evaluate the effect of different osteoconductive bone substitutes on the bone repair in critical-sized defects (CSDs) in rat calvaria.

Material and method: One CSD with an $8 \mathrm{~mm}$ diameter was made in each of the 40 rats used in this study. The animals were randomly allocated into 5 groups $(n=8)$, according to the type of bone substitute used to fill the CSD: COA (Coagulum); AUT (autogenous bone); DBB (deproteinized bovine bone graft); HA/TCP (biphasic ceramic composed of hydroxyapatite and $\beta$-phosphate tricalcium); and TCP ( $\beta$-phosphate tricalcium). A microtomographic analysis was performed to evaluate the remaining defect linear length (DLL) of the CSD and the volume of the mineralized tissues (MT) within the CSD at 3, 7, 15 and 30 days after the surgical procedure. In addition, a histometric analysis was performed to evaluate the composition of the repaired bone tissue (\% Bone and \% Biomaterial) at the 30-day period.

Result: It was shown that the COA had the lowest DLL and MT within the CSD. In addition, the COA presented the highest \% of bone in CSD. The DBB had a higher MT and a higher \% of bone substitute particles in the CSD than the AUT and TCP groups. The DBB and AUT groups presented higher \% of bone in the CSD than the TCP group.

Conclusion: The use of the DBB promoted a better pattern of bone volume gain and formation compared to TCP and HA / TCP but was biologically inferior to the AUT.

Descriptors: Histology; x-ray microtomography; bone regeneration; bone substitutes.

Instituição afiliada: 1- UNESP - Universidade Estadual Paulista, Faculdade de Odontologia de Araraquara, Departamento de Diagnóstico e Cirurgia, Araraquara, SP, Brasil. 2- UFU - Universidade Federal de Uberlândia, Departamento de Periodontia, Uberlândia, SP, Brasil

Dados da publicação: Artigo recebido em 01 de agosto, revisado em 10 de agosto, aceito para publicação em 19 de agosto e publicado em 29 de Agosto.

DOI: https://doi.org/10.36557/2674-8169.2020v2n9p28-44

@ Elcio Marcantonio Junior elcio.marcantonio@unesp.br 


\section{Reparo ósseo induzido por diferentes substitutos de enxerto ósseo em defeitos de tamanho crítico na calvária de ratos. \\ Aroni et al.}

\section{INTRODUÇÃO}

A regeneração de defeitos ósseos causados por diferentes agentes etiológicos continua sendo um grande desafio nas áreas médica e odontológica ${ }^{1-3}$. Embora o enxerto ósseo autógeno (AUT) seja considerado o padrão ouro para técnicas de aumento da disponibilidade óssea ${ }^{4-7}$, o uso desse enxerto apresenta algumas limitações, como a morbidade do leito doador, o aumento do tempo cirúrgico, a quantidade limitada de enxerto disponível e a taxas de reabsorção elevadas, principalmente quando utilizadas na forma particulada ${ }^{5}$.

Essas limitações têm indicado a necessidade da utilização de outros tipos de substitutos ósseos que permitam um processo adequado de formação do tecido ósseo com redução da morbidade ao hospedeiro ${ }^{8,9}$. Dentre as alternativas de substituto ósseo ao enxerto ósseo autógeno, biomateriais osteocondutores, como osso bovino desproteinizado (DBB) e cerâmicas bifásicas, que são compostas por hidroxiapatita e beta-fosfato tricálcico (HA / TCP), têm aplicação clínica altamente difusa ${ }^{8-10}$. No entanto, a baixa taxa de reabsorção da hidroxiapatita presente em ambos os biomateriais implica em sua substituição completa por tecido ósseo, sendo que os remanescentes desses substitutos ósseos foram observados no local receptor até a colocação do implante ${ }^{9,10}$. Além disso, essas partículas remanescentes não participam do processo de osseointegração direta dos implantes devido à falta de vitalidade desse tecido ${ }^{11}$.

Outro biomaterial com propriedades osteocondutoras e amplamente utilizado como substituto ósseo é o $\beta$-fosfato tricálcico (TCP). O TCP apresentou resultados clínicos na elevação do seio maxilar semelhantes ao $\mathrm{DBB}^{12,13}$ e $\mathrm{AUT}^{6}$. Em contraste com outros substitutos ósseos osteocondutores, O TCP tem sido relacionado a maiores taxas de reabsorção, o que pode indicar que esse substituto ósseo pode induzir uma maior quantidade de osso novo na área enxertada ${ }^{7}$.

A comparação direta entre os diferentes substitutos ósseos osteocondutores tem sido pouco explorada. Um estudo pré-clínico comparou o potencial de reparo ósseo de TCP com DBB, AUT e coágulo (COA) em defeitos de tamanho crítico (DAGs) em calvária de rato $\stackrel{7}{\text {. Neste }}$ estudo, O TCP induziu o reparo ósseo com característica biológica semelhante às áreas enxertadas com DBB, porém com menor formação óssea que o AUT. No entanto, os períodos de avaliação neste estudo foram superiores a 30 dias e não foi avaliada alteração volumétrica do tecido reparado em períodos anteriores. Além disso, a comparação direta dos principais substitutos ósseos osteocondutores (DBB, HA / TCP e TCP) com osso autógeno não foi previamente realizada longitudinalmente no mesmo animal.

Assim, o objetivo deste estudo foi avaliar a influência de diferentes substitutos do enxerto ósseo (DBB, HA / TCP e TCP) no reparo da DSC por meio de análise microtomográfica (Análise do comprimento linear do defeito remanescente e volume de tecido mineralizado nos estágios iniciais de consolidação óssea) e análise histológica (Análise da composição do 
tecido enxertado). A hipótese nula deste estudo é que os diferentes substitutos ósseos osteocondutores não apresentarão diferenças no reparo da DSC.

\section{MATERIAL E MÉTODO}

O estudo foi aprovado pelo Comitê de Ética para Uso de Animais de nossa instituição (19/2014) e foi conduzido de acordo com os princípios orientadores internacionais para pesquisas biomédicas envolvendo animais e as diretrizes ARRIVE. No presente estudo, foram usados 40 ratos adultos machos (Rattus Norvegicus, albinus var., Holtzman) com massas corporais variando de 300-350 gramas. Os animais foram mantidos em ambiente com luz e temperatura controladas, alimentados com ração sólida e tiveram acesso a água ad libitum antes e durante o período experimental.

\section{Grupos}

Os 40 animais foram alocados aleatoriamente, por lote após a confecção do CSD, em 5 grupos de 8 animais cada: Grupo COA - O CDS foi preenchido com coágulo; AUT - O DSC foi preenchido com enxerto ósseo autógeno obtido durante a confecção do DSC; Grupo DBB - O DSC foi preenchido com enxerto ósseo bovino desproteinizado (Bio-Oss ${ }^{\circledR}$, Geistlich AG, Wolhusen, Suíça); Grupo HA / TCP - O CSD foi preenchido com cerâmica bifásica composta por hidroxiapatita e $\beta$-fosfato tricálcico (Straumann ${ }^{\circledR}$ Bone Ceramic, Straumann AG, Basel, Suíça); e grupo TCP - O CSD foi preenchido $\operatorname{com} \beta$ fosfato tricálcico (Beta Pro ${ }^{\circledR}$, Procell, São Carlos, Brasil).

Para o cálculo do tamanho da amostra, os dados de formação óssea nos DSC que foram preenchidos com DBB e TCP e foram avaliados pela análise histométrica de Silva et al. $\stackrel{7}{\text { foram }}$ usados. A diferença mínima de médias entre os grupos em que foram encontradas diferenças significativas foi de $27,9 \%$ com desvio padrão de $9,0 \%$. Assim, ao estabelecer o poder $\beta$ de 0,90 e o erro $\alpha$ de 0,05, foi determinado um número mínimo de 6 animais por grupo. Utilizamos mais dois animais por grupo para evitar a perda do poder estatístico devido à perda de animais durante o período experimental.

\section{Procedimento Cirúrgico}

Os animais foram anestesiados com uma combinação de cetamina e xilazina na proporção de $0,08 \mathrm{ml} / \mathrm{kg}$ de peso corporal de cetamina (Cloridrato de Cetamina, Francotar 10\%, Virbac do Brasil Ind. Com. Ltda, São Paulo, Brasil) associada a 0,04 ml / kg de peso corporal de xilazina (Cloridrato de Xilazina - Virbaxil 2\% - Virbac do Brasil Ind. E Com. Ltda., São Paulo, Brasil) e posteriormente submetidos à tricotomia na região da calvária. A antissepsia do campo cirúrgico foi então realizada com solução de iodopovidona. 
O acesso cirúrgico à calvária foi obtido através de incisão bicoronal cutânea e muscular, o que permitiu a exposição do tecido ósseo. Em seguida, os tecidos foram divulsionados até a exposição do periósteo, que foi incisado e descolado, para que fosse obtida a exposição do tecido ósseo.

Logo após o ápice da sutura coronal do animal foi confeccionado defeito ósseo circular com broca trefina de diâmetro externo de $8 \mathrm{~mm}$ (3i - 3i implantes do Brasil, São Paulo, Brasil). A seguir, o DSC apresentava-se com diâmetro de $8 \mathrm{~mm}$ e espessura aproximada de 1,5 $\mathrm{mm}$, dado pela retirada total do tecido ósseo. Os CSDs foram realizados com o auxílio de um contra-ângulo (Anthogyr, Injecta, Diadema, Brasil) com redução de 16: 1 acoplado a uma rotação do motor de 1500 rpm (BML 600 Plus Driller, CK Driller, Cacapicuíba, Brasil), sob irrigação constante com solução salina. Todos os DSC foram feitos pelo mesmo cirurgião (MATA).

Cada biomaterial foi implantado no DSC até o seu preenchimento total sem extravasamento. Em seguida, todos os defeitos foram recobertos por membrana de colágeno (Genius-Baumer, Mogi-Mirim, Brasil) e suturados com fio de seda 4.0 (Ethicon, Johnson \& Johnson, São José dos Campos, Brasil). Os animais foram tratados no pós-operatório com um único multibiótico (Pentabiotico veterinario veterinária, Zoetis Dodge, São Paulo, Brasil, Subcutâneo: 0,03 ml / kg) e uma dose de cetoprofeno (Ketoflex; Mundo Animal, São Paulo, Brasil, Intramuscular: 0,03 ml / kg).

\section{Análise Micro CT}

Os animais foram anestesiados para serem submetidos à varredura aos 3, 7, 15 e 30 dias após o procedimento cirúrgico em micro-tomógrafo (SkyScan, Kontich, Bélgica). Os parâmetros utilizados para a digitalização foram os seguintes: Pixel da câmera: 12,45; Tensão: $65 \mathrm{Kv}$; Amperagem: $385 \mu \mathrm{A}$; e Filtro: Alumínio $1 \mathrm{~mm}$. As imagens geradas foram posteriormente reconstruídas, reorientadas espacialmente e analisadas por um software específico (NRecon / DataViewer / CTan, Skyscan, Aartselaar, Bélgica). Duas análises foram realizadas: 1) análise do comprimento linear remanescente (RLL) do DSC que foi medido na maior porção do DSC nos planos sagital e coronal, e 2) análise do volume dos tecidos mineralizados (MT) dentro do DSC, que foi realizado dentro de uma região circular de interesse (ROI), de $8 \mathrm{~mm}$ de diâmetro com espessura de 30 cortes axiais com espessura de 18 $\mu \mathrm{m}$. O tecido ósseo associado aos substitutos ósseos foi detectado pela aplicação de um limite dentro do intervalo de 55-250 na escala de cinza dentro da ROI. A análise da micro TC foi realizada por um avaliador cego, treinado e calibrado (GJO).

\section{Colheita de amostras cirúrgicas e análise histológica / histométrica}

Após a realização da micro tomografia computadorizada no período de 30 dias, os animais foram eutanasiados por overdose de anestésico. Em seguida, as regiões da calvária relacionadas aos defeitos foram retiradas e fixadas em paraformaldeído $4 \%$ por 48 horas e 
descalcificadas em EDTA 7\% por 3 meses para posterior execução de processamento laboratorial para inclusão das amostras em parafina e obtenção de cortes histológicos de 5 $\mu \mathrm{m}$ de espessura que foram corados pela técnica de hematoxilina e eosina.

Posteriormente, três seções histológicas equidistantes (50 $\mu \mathrm{m}$ de distância) de cada animal foram fotografadas usando uma câmera (Leica Microsystems DFC-300-FX; Leica Reichert \& Jung Products, Wetzlar, Alemanha), que foi acoplada sob um microscópio óptico (DM2500, Leica Produtos Reichert \& Jung, Wetzlar, Alemanha) com uma ampliação de 25x.

$\mathrm{Na}$ análise histométrica, o DSC original foi delimitado com base nas linhas de reversão que marcavam o defeito original e, posteriormente, os diferentes componentes dentro da área total do defeito original (osso neoformado e remanescentes de enxerto ósseo) foram medidos por analisador de imagem de software (ImageJ, Jandel Scientific, San Rafael, CA, EUA), e esses valores foram dados em porcentagem, em relação à área total. As análises histométricas foram realizadas por avaliador cego e treinado para essas análises (MATA).

\section{Análise Estatística}

O software GraphPad Prism 6 (San Diego, CA, EUA) foi usado para realizar a análise estatística. Os dados histológicos e microtomográficos tiveram distribuição normal confirmada pelo teste de normalidade Shapiro-Wilk. A avaliação comparativa entre os diferentes grupos foi realizada por meio dos testes paramétricos de ANOVA one-way complementados pelo teste de Tukey. A avaliação dentro de cada grupo comparando diferentes períodos experimentais foi realizada por meio dos testes paramétricos do teste ANOVA de medidas repetidas complementados pelo teste de Tukey. Todos os testes estatísticos deste estudo foram aplicados ao nível de significância de 5\%.

Este estudo é uma adaptação para o português do artigo original " Aroni, Mauricio Andrés Tinajero, et al. "Bone repair induced by different bone graft substitutes in critical-sized defects in rat calvaria." Revista de Odontologia da UNESP 48 (2019). 29 " E segue os preceitos Creative Commons - Atribuição 4.0 Internacional - CC BY 4.0 de livre distribuição, uso ou remixagem. Nenhum dos autores endossou este compartilhamento.

\section{RESULTADO}

Todos os animais sobreviveram aos procedimentos cirúrgicos e permaneceram saudáveis durante todo o período experimental. Para avaliação das calibrações dos examinadores, 10 varreduras microtomográficas e 30 lâminas histológicas foram medidas em duplicata com intervalo de uma semana. A correlação de Pearson mostrou índice $r>0,89$ para os examinadores em todos os parâmetros avaliados.

\section{Análise Micro CT}




\section{Reparo ósseo induzido por diferentes substitutos de enxerto ósseo em defeitos de tamanho crítico na calvária de ratos. \\ Aroni et al.}

Todos os grupos apresentaram redução progressiva do RLL em períodos mais longos de avaliação. Também foi mostrado que o grupo COA $(6,34 \pm 1,49 \mathrm{~mm})$ teve um RLL menor em comparação com os grupos DBB $(7,64 \pm 0,41 \mathrm{~mm}), \operatorname{TCP} / \mathrm{HA}(7,55 \pm 0,39 \mathrm{~mm})$ e TCP $(7,59$ $\pm 0,33 \mathrm{~mm}$ ) no Período de 3 dias $(p<0,05)$. Além disso, o grupo COA apresentou menor RLL em relação ao grupo $\mathrm{DBB}(5,80 \pm 1,24 \mathrm{~mm}$ vs. $7,01 \pm 0,38 \mathrm{~mm})$ no período de 30 dias $(p<0,05)$ ( Tabela 1 ).

Tabela 1 A média e o desvio padrão dos dados RLL $(\mathrm{mm})$ em todos os grupos e períodos de avaliação

\begin{tabular}{|c|c|c|c|c|}
\hline Grupo / Período & 3 dias & 7 dias & 15 dias & 30 dias \\
\hline \multirow{2}{*}{$\mathrm{COA}$} & $\div 8$ & $B$ & $B$ & $\bar{A}$ \\
\hline & $6,34 \pm 1,49^{-}$ & $6,41 \pm 0,63$ & $6,48 \pm 0,93$ & $5,80 \pm 1,24 \#$ \\
\hline \multirow{2}{*}{ AUT } & 8 & $A B$ & A & A \\
\hline & $7,37 \pm 0,67$ & $7,17 \pm 0,83$ & $6,65 \pm 0,72$ & $6,27 \pm 0,62$ \\
\hline \multirow{2}{*}{ DBB } & 8 & $A B$ & $A B$ & A \\
\hline & $7,64 \pm 0,41$ & $7,31 \pm 0,86$ & $7,29 \pm 0,36$ & $7,01 \pm 0,38$ \\
\hline \multirow{2}{*}{$\mathrm{TCP} / \mathrm{HA}$} & B & A & A & A \\
\hline & $7,55 \pm 0,39$ & $6,85 \pm 0,45$ & $6,82 \pm 0,51$ & $6,53 \pm 0,53$ \\
\hline \multirow{2}{*}{ TCP } & $B$ & $A B$ & A & A \\
\hline & $7,59 \pm 0,33$ & $6,94 \pm 0,51$ & $6,84 \pm 0,70$ & $6,85 \pm 0,71$ \\
\hline
\end{tabular}

RLL mais curto em comparação com os grupos DBB, TCP / HA e TCP no período de 3 dias; "RLL mais curto do que o grupo DBB no período de 30 dias. ANOVA de uma via complementada pelo teste de Tukey $(p<0,05)$. Letras diferentes representam diferenças estatísticas intragrupo - ANOVA de medidas repetidas complementada pelo teste de Tukey.

Em relação ao volume de $\mathrm{MT}$, verificou-se que o grupo COA sempre apresentou valores inferiores desse parâmetro (5,17 - 28,04\%) em relação aos demais grupos ( $>42,77 \%)$ em todos os períodos de avaliação $(p<0,05)$. O grupo DBB $(65,66 \pm 3,95 \%)$ apresentou maior volume de MT do que o grupo AUT $(42,77 \pm 6,12 \%)$ no período de 7 dias $(p<0,05)$. Além disso, o grupo DBB $(59,05 \pm 6,13 \%)$ apresentou maior volume de MT do que os grupos AUT $(44,93 \pm 5,72 \%)$ e TCP $(45,16 \pm 5,03 \%)$ no período de 15 dias $(p<0,05)$ ( Tabela 2 ) Comparando os diferentes períodos experimentais, notou-se uma redução progressiva no volume da TM em todos os grupos, exceto no grupo COA que apresentou melhora progressiva neste parâmetro em períodos mais longos de avaliação. figura 1 mostra as imagens representativas da análise microtomográfica de todos os grupos.

\section{Histometria}

A análise histométrica foi realizada apenas no período experimental de 30 dias. Verificou-se que o grupo COA apresentou maior quantidade de osso neoformado do que todos os outros grupos $(17,42 \pm 0,68 \%)$. Além disso, os grupos DBB $(8,39 \pm 0,71 \%)$ e AUT $(7,96$ $\pm 0,79 \%)$ apresentaram mais osso neoformado do que o grupo TCP $(6,32 \pm 0,80 \%)$. Além disso, o grupo DBB $(24,21 \pm 1,44 \%)$ apresentou maior remanescente de enxerto ósseo do que os grupos AUT $(17,48 \pm 0,72 \%)$ e TCP $(17,17 \pm 0,74 \%)$. O grupo TCP / HA não apresentou 
diferenças significativas em relação aos demais grupos de biomateriais na quantidade de osso neoformado $(7,22 \pm 0,76 \%)$, bem como na quantidade de remanescentes de enxerto ósseo $(22,78 \pm 0,90 \%$ ) ( Tabela 3 ) A Figura 2 mostra as imagens histológicas representativas de cada grupo.

Tabela 2 A média e o desvio padrão dos dados de volume de MT (\%) em todos os grupos e períodos de avaliação

\begin{tabular}{|c|c|c|c|c|}
\hline Grupo / Período & 3 dias & 7 dias & 15 dias & 30 dias \\
\hline \multirow[b]{2}{*}{$\mathrm{COA}$} & ${ }^{\circ} \mathrm{C}$ & ${ }^{* B}$ & $\cdot A$ & $\cdot A$ \\
\hline & $5,12 \pm 2,48$ & $18,92 \pm 3,58$ & $28,04 \pm 3,90$ & $27,54 \pm 4,66$ \\
\hline \multirow{2}{*}{ AUT } & A & $\# B$ & $B$ & $B$ \\
\hline & $63,62 \pm 3,86$ & $42,77 \pm 6,12$ & $44,93 \pm 5,726$ & $46,82 \pm 4,21$ \\
\hline \multirow{2}{*}{ DBB } & A & A & $B$ & $B$ \\
\hline & $72,14 \pm 4,72$ & $65,66 \pm 3,95$ & $59,05 \pm 6,13$ & $51,43 \pm 3,85$ \\
\hline \multirow{2}{*}{$\mathrm{TCP} / \mathrm{HA}$} & A & $B$ & $B$ & $\mathrm{C}$ \\
\hline & $68,37 \pm 3,90$ & $54,48 \pm 4,33$ & $47,09 \pm 7,52$ & $44,12 \pm 2,97$ \\
\hline \multirow{2}{*}{ TCP } & A & $B$ & $6 B$ & $B$ \\
\hline & $66,80 \pm 7,31$ & $50,71 \pm 3,61$ & $45,16 \pm 5,03$ & $43,81 \pm 4,00$ \\
\hline
\end{tabular}

Menor volume de MT do que todos os outros grupos em todos os períodos experimentais; \# Menor volume de MT do que o grupo DBB no período de 7 dias; $\delta$ Volume de MT menor do que o grupo DBB no período de 15 dias. ANOVA de uma via complementada pelo teste de Tukey $(p<0,05)$. Letras diferentes representam diferenças estatísticas intragrupo - ANOVA de medidas repetidas complementada pelo teste de Tukey.

Tabela 3 A média e o desvio padrão dos dados da composição do tecido reparado dentro do CSD (\% osso neoformado e\% remanescentes de enxerto ósseo) determinados por análise histométrica

\begin{tabular}{|c|c|c|}
\hline Grupo / Tecido & $\%$ Osso recém-formado & $\%$ Restos de enxerto ósseo \\
\hline $\mathrm{COA}$ & $15,95 \pm 0,91^{a}$ & - \\
\hline AUT & $10,74 \pm 0,81$ & $17,48 \pm 0,72^{b}$ \\
\hline DBB & $8,39 \pm 0,71^{c}$ & $24,21 \pm 1,44^{a}$ \\
\hline $\mathrm{TCP} / \mathrm{HA}$ & $7,22 \pm 0,76^{c, d}$ & $22,78 \pm 0,90^{a}$ \\
\hline TCP & $6,32 \pm 0,80^{d}$ & $17,17 \pm 0,74^{b}$ \\
\hline
\end{tabular}

Letras diferentes representam diferentes niveis de diferenças significativas entre os grupos - ANOVA de uma via complementada pelo teste de Tukey $(p<0,05)$. 

crítico na calvária de ratos.

Aroni et al.

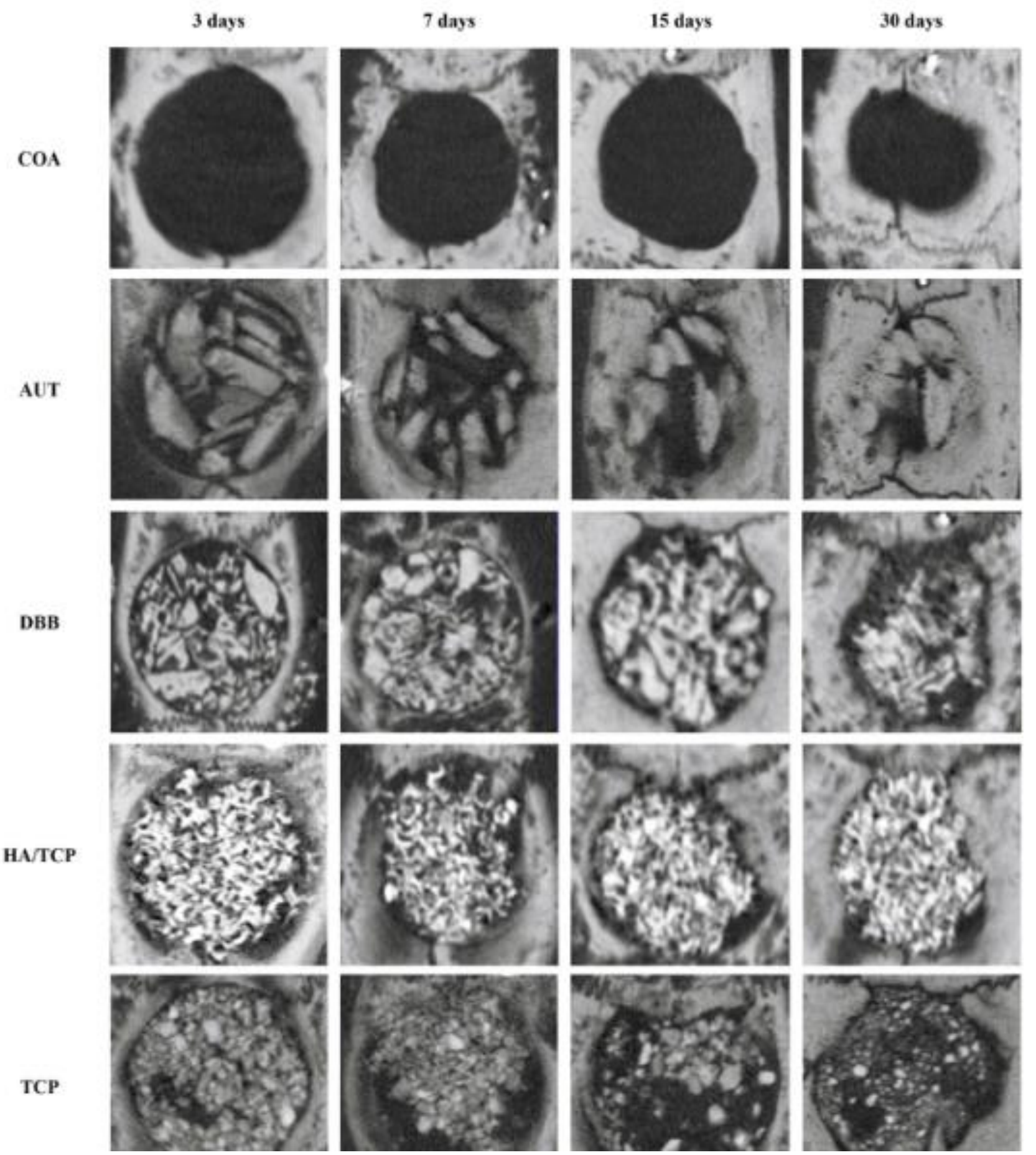

Figura 1: Imagens representativas de micro TC. É possível observar que os grupos onde o DSC foi preenchido com biomateriais apresentaram mais TM do que o grupo COA; entretanto, este subgrupo apresenta um maior fechamento do DSC em relação aos demais grupos, com exceção do grupo AUT. 


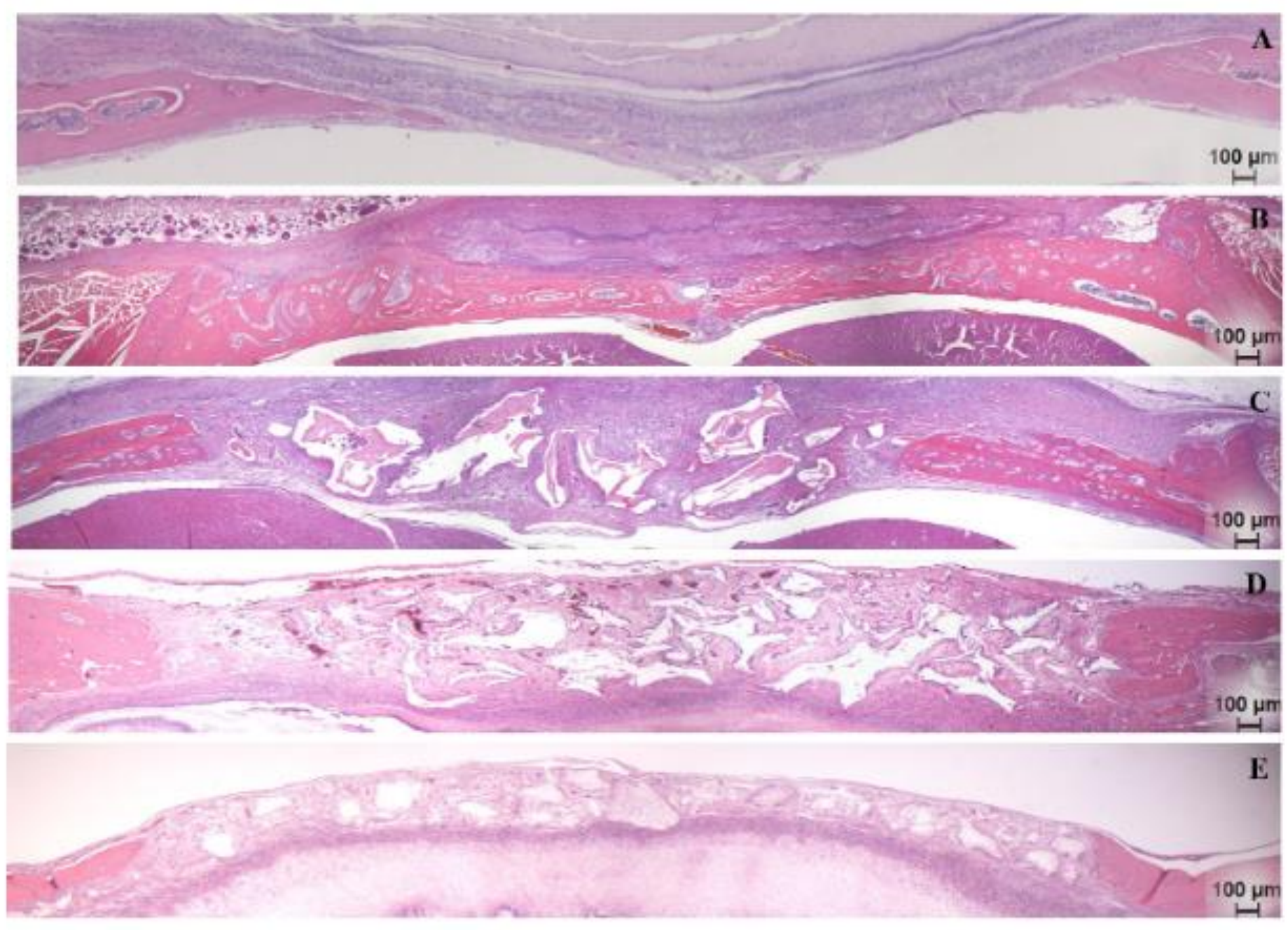

Figura 2 Imagens histológicas representativas de todos os grupos. A) COA; B) AUT; C) DBB; D) HA / TCP; E) TCP. Apesar do menor ganho volumétrico, o grupo COA apresenta maior osso neoformado do que os grupos DBB, HA / TCP e TCP. O grupo AUT apresentou a melhor associação de formação óssea e aumento do volume ósseo. Em todos os grupos, a formação óssea iniciouse nas bordas e avançou em direção ao centro do DSC (original magnification -25X-coloração HE).

\section{DISCUSSÃO}

Este estudo demonstrou que os diferentes substitutos ósseos osteocondutores testados neste estudo (DBB, HA / TCP e TCP) promoveram um aumento volumétrico de MT dentro do CSD que foi associado à formação de tecido ósseo e à presença de restos de partículas de biomateriais que estiveram em contato com o osso neoformado, principalmente nas bordas do DSC, o que confirma as propriedades osteocondutoras desses substitutos ósseos. Entretanto, a formação óssea nesses grupos foi menor do que a observada nos grupos COA e AUT.

O grupo COA apresentou maior fechamento do DSC em comparação aos grupos onde os substitutos ósseos osteocondutores foram utilizados para preenchimento do DSC já no 
período de 3 dias, e esse achado foi associado à maior formação óssea observada também nos grupos COA . Estudos pré-clínicos demonstraram que o preenchimento de alvéolos pósextração com biomateriais reduz a formação de tecido ósseo em comparação com os alvéolos pós-extração que foram mantidos sem biomateriais durante o período de cicatrização ${ }^{14-16}$. Esse fenômeno se deve à redução do espaço para a formação óssea por ser ocupado por restos de biomateriais ${ }^{16}, 17$, evento confirmado por este estudo. Além disso, 0 coágulo apresenta fatores de crescimento que aceleram a regeneração óssea $\underline{18}, \underline{19}$. Porém, vale ressaltar que o grupo COA apresentou menor volume de $\mathrm{MT}$, o que demonstra que o uso de biomateriais é importante para se obter disponibilidade suficiente de tecido de reparo para manter ou melhorar a morfologia óssea ${ }^{14,20}$.

O DSC preenchido com AUT apresentou fechamento semelhante ao grupo COA e maior formação óssea do que o DSC preenchido com HA / TCP e TCP, bem como menos restos de biomateriais que o DSC preenchido com DBB. Esses dados, em conjunto, mostram que a área reparada com o uso do AUT apresentou as melhores características biológicas entre os grupos avaliados, confirmando sua posição como substituto ósseo padrão ouro por ser a única que apresenta as propriedades biológicas de formação óssea como osteoindução, osteogênese e osteocondução ${ }^{4,7}$.

Dentre os substitutos ósseos testados neste estudo, o grupo DBB apresentou maior quantidade de volume de MT do que os grupos AUT e TCP, e esse achado foi relacionado à maior quantidade de restos de partículas DBB. Este fato confirma os achados de outros estudos que mostraram que o componente hidroxiapatita bovina é menos sujeito a reabsorção do que os demais substitutos ósseos ${ }^{21}$. A quantidade de partículas de biomaterial e formação óssea nos grupos HA / TCP foi semelhante ao grupo DBB, embora não tenha diferido dos grupos AUT e TCP em termos de volume de MT e quantidade de biomaterial remanescente. O mecanismo de ação do HA / TCP é baseado na reabsorção do componente TCP que promoveria a diferenciação das células mesenquimais nos osteoblastos e na manutenção do componente HA que serviria de arcabouço para a formação óssea em contato próximo com este componente $8,20,22$. A associação do TCP, que é mais reabsorvível com HA, que é pouco reabsorvível, pode ser a justificativa para que as cerâmicas bifásicas de HA / TCP não tenham se diferenciado dos demais biomateriais osteocondutores testados neste estudo.

Por outro lado, o grupo TCP apresentou menores volumes de MT do que o grupo DBB no período de 15 dias, menor quantidade de substituto ósseo remanescente do que os grupos DBB e HA / TCP e menor quantidade de osso neoformado que o DBB e Grupos AUT. Esse achado está de acordo com estudos anteriores que demonstraram que o TCP apresenta maiores taxas de reabsorção do que o HA ${ }^{22,23}$. Como o TCP mantém menos espaço que o $\mathrm{HA}$, é provável que o torne menos competente em termos de manutenção volumétrica da área enxertada. A maior taxa de reabsorção em relação ao $\mathrm{DBB}^{23,24}$, e as menores propriedades biológicas de formação óssea em relação ao enxerto ósseo autógeno ${ }^{7,25}$, pode justificar a formação óssea inferior mostrada no grupo TCP. 


\section{Reparo ósseo induzido por diferentes substitutos de enxerto ósseo em defeitos de tamanho crítico na calvária de ratos. \\ Aroni et al.}

Os resultados deste estudo devem ser interpretados com cautela, pois a superioridade de um biomaterial em relação ao outro não significa que clinicamente essa diferença tenha alguma relevância de forma que possa diferenciar taxas de sobrevivência e sucesso de implantes em áreas enxertadas com esses biomateriais. De fato, implantes colocados em áreas enxertadas com DBB, HA / TCP e TCP apresentam altos níveis de sobrevivência e sucesso $\underline{6}, \underline{26}, \underline{27}$. Assim, pode ser possível que implantes sejam colocados mais precocemente em áreas enxertadas com osso autógeno, DBB ou HA / TCP em comparação com áreas enxertadas com TCP, porém esta hipótese deve ser testada em outros estudos.

Este estudo apresenta limitações que devem ser levadas em consideração na interpretação de nossos resultados. Os resultados dos estudos pré-clínicos não apresentarão necessariamente o mesmo padrão em humanos, portanto, as inferências clínicas baseadas nos dados deste estudo são limitadas. Além disso, o tipo de defeito avaliado foi o crítico e a resposta em defeitos não críticos como em seio maxilar e alvéolos pós-extração apresenta comportamento biológico diferente que pode alterar a cicatrização promovida pelos diferentes substitutos ósseos avaliados neste estudo. Apesar dessas limitações, vale ressaltar que os DSC têm sido um modelo amplamente utilizado para avaliação da consolidação óssea em áreas enxertadas. O DSC de $8 \mathrm{~mm}$ usado neste estudo tem potencial regenerativo limitado com boa previsibilidade de ausência de fechamento completo do defeito, que é característico de defeitos críticos. Portanto, este modelo representa uma condição desafiadora para avaliar o potencial regenerativo dos diferentes substitutos ósseos ${ }^{28}$.

Pode-se concluir que o DBB promoveu um melhor padrão de volume de MT e quantidade de osso neoformado em comparação ao TCP e HA / TCP, porém com menor formação óssea e maior remanescente de partículas do que o grupo AUT. Devido a isso, a hipótese nula deste estudo foi rejeitada.

\section{REFERÊNCIAS}

1 Freitas RM, Susin C, Tamashiro WM, Souza JAC, Marcantonio C, Wikesjö UM, et al. Histological analysis and gene expression profile following augmentation of the anterior maxilla using rhBMP-2/ACS versus autogenous bone graft. J Clin Periodontol. 2016 Dec;43(12):1200-7. http://dx.doi.org/10.1111/jcpe.12601. PMid:27440671. [ Links ]

2 Friedrich JB, Moran SL, Bishop AT, Shin AY. Free vascularized fibula grafts for salvage of failed oncologic long bone reconstruction and pathologic fractures. Microsurgery. 2009;29(5):385-92. http://dx.doi.org/10.1002/micr.20624. PMid:19296529. [ Links ]

3 Hanke A, Bäumlein M, Lang S, Gueorguiev B, Nerlich M, Perren T, et al. Long-term radiographic appearance of calcium-phosphate synthetic bone grafts after surgical treatment of tibial plateau fractures. Injury. 2017 Dec;48(12):2807-

13. http://dx.doi.org/10.1016/i.injury.2017.10.030. PMid:29096930. [ Links ] 
4 Spin-Neto R, Stavropoulos A, Coletti FL, Faeda RS, Pereira LA, Marcantonio E Jr. Graft incorporation and implant osseointegration following the use of autologous and fresh-frozen allogeneic block bone grafts for lateral ridge augmentation. Clin Oral Implants Res. 2014 Feb;25(2):226-33. http://dx.doi.org/10.1111/clr.12107. PMid:23346871. [ Links ]

5 Nkenke E, Neukam FW. Autogenous bone harvesting and grafting in advanced jaw resorption: morbidity, resorption and implant survival. Eur J Oral Implantol. 2014;7(Suppl 2):S203-17. PMid:24977256. [ Links ]

6 Zijderveld SA, Zerbo IR, van den Bergh JP, Schulten EA, ten Bruggenkate CM. Maxillary sinus floor augmentation using a beta-tricalcium phosphate (Cerasorb) alone compared to autogenous bone grafts. Int J Oral Maxillofac Implants. 2005 May-Jun;20(3):432-40. PMid:15973955. [ Links ]

7 Silva LF, Reis ENRC, Barbara TA, Bonardi JP, Garcia IR, Carvalho PSP, et al. Assessment of bone repair in critical-size defect in the calvarium of rats after the implantation of tricalcium phosphate beta ( $\beta$-TCP). Acta Histochem. 2017 Jul;119(6):624-

31. http://dx.doi.org/10.1016/j.acthis.2017.07.003. PMid:28732677. [ Links ]

8 Cordaro L, Bosshardt DD, Palattella P, Rao W, Serino G, Chiapasco M. Maxillary sinus grafting with Bio-Oss or Straumann Bone Ceramic: histomorphometric results from a randomized controlled multicenter clinical trial. Clin Oral Implants Res. 2008 Aug;19(8):796803. http://dx.doi.org/10.1111/j.1600-0501.2008.01565.x. PMid:18705811. [ Links ]

9 Wang F, Zhou W, Monje A, Huang W, Wang Y, Wu Y. Influence of healing period upon bone turn over on maxillary sinus floor augmentation grafted solely with deproteinized bovine bone mineral: a prospective human histological and clinical trial. Clin Implant Dent Relat Res. 2017 Apr;19(2):341-50. http://dx.doi.org/10.1111/cid.12463. PMid:27862924. [ Links ]

10 Uzeda MJ, de Brito Resende RF, Sartoretto SC, Alves ATNN, Granjeiro JM, Calasans-Maia MD. Randomized clinical trial for the biological evaluation of two nanostructured biphasic calcium phosphate biomaterials as a bone substitute. Clin Implant Dent Relat Res. 2017 Oct;19(5):802-11. http://dx.doi.org/10.1111/cid.12516. PMid:28703478. [ Links ]

11 Carmagnola D, Abati S, Celestino S, Chiapasco M, Bosshardt D, Lang NP. Oral implants placed in bone defects treated with Bio-Oss, Ostim-Paste or PerioGlas: an experimental study in the rabbit tibiae. Clin Oral Implants Res. 2008 Dec;19(12):1246-

53. http://dx.doi.org/10.1111/j.1600-0501.2008.01584.x. PMid:19040439. [ Links ]

12 Martinez A, Franco J, Saiz E, Guitian F. Maxillary sinus floor augmentation on humans: Packing simulations and 8 months histomorphometric comparative study of anorganic bone matrix and $\beta$-tricalcium phosphate particles as grafting materials. Mater Sci Eng C Mater Biol Appl. 2010 Jun;30(5):763-9. http://dx.doi.org/10.1016/j.msec.2010.03.012. PMid:21625341. [ Links ] 


\section{Reparo ósseo induzido por diferentes substitutos de enxerto ósseo em defeitos de tamanho crítico na calvária de ratos. \\ Aroni et al.}

13 Trombelli L, Franceschetti G, Stacchi C, Minenna L, Riccardi O, Di Raimondo R, et al. Minimally invasive transcrestal sinus floor elevation with deproteinized bovine bone or $\beta$ tricalcium phosphate: a multicenter, double-blind, randomized, controlled clinical trial. J Clin Periodontol. 2014 Mar;41(3):311-9. http://dx.doi.org/10.1111/jcpe.12210. PMid:24325663. [ Links ]

14 Araújo MG, Lindhe J. Ridge preservation with the use of Bio-Oss collagen: a 6-month study in the dog. Clin Oral Implants Res. 2009 May;20(5):433-

40. http://dx.doi.org/10.1111/j.1600-0501.2009.01705.x. PMid:19522974. [ Links ]

15 Araújo MG, Sonohara M, Hayacibara R, Cardaropoli G, Lindhe J. Lateral ridge augmentation by the use of grafts comprised of autologous bone or a biomaterial. An experiment in the dog. J Clin Periodontol. 2002 Dec;29(12):1122-

31. http://dx.doi.org/10.1034/i.1600-051X.2002.291213.x. PMid:12492915. [ Links ]

16 Carmagnola D, Adriaens $P$, Berglundh T. Healing of human extraction sockets filled with Bio-Oss. Clin Oral Implants Res. 2003 Apr;14(2):137-43. http://dx.doi.org/10.1034/j.16000501.2003.140201.x. PMid:12656871. [ Links ]

17 Arruda T, Sukekava F, de Souza AB, Rasmusson L, Araújo MG. Early healing in alveolar sockets grafted with titanium granules. An experimental study in a dog model. J Biomed Mater Res A. 2013 Jul;101(7):1971-6. http://dx.doi.org/10.1002/jbm.a.34501.

PMid:23225833. [ Links ]

18 Wang X, Friis TE, Masci PP, Crawford RW, Liao W, Xiao Y. Alteration of blood clot structures by interleukin-1 beta in association with bone defects healing. Sci Rep. 2016 Oct;6:35645. http://dx.doi.org/10.1038/srep35645. PMid:27767056. [ Links ]

19 Yang J, Zhou Y, Wei F, Xiao Y. Blood clot formed on rough titanium surface induces early cell recruitment. Clin Oral Implants Res. 2016 Aug;27(8):1031-

8. http://dx.doi.org/10.1111/clr.12672. PMid:26332946. [ Links ]

20 Lindhe J, Araújo MG, Bufler M, Liljenberg B. Biphasic alloplastic graft used to preserve the dimension of the edentulous ridge: an experimental study in the dog. Clin Oral Implants Res. 2013 Oct;24(10):1158-63. http://dx.doi.org/10.1111/j.1600-0501.2012.02527.x.

PMid:22804845. [ Links ]

21 Kato E, Lemler J, Sakurai K, Yamada M. Biodegradation property of beta-tricalcium phosphate-collagen composite in accordance with bone formation: a comparative study with Bio-Oss Collagen in a rat critical-size defect model. Clin Implant Dent Relat Res. 2014 Apr;16(2):202-11. http://dx.doi.org/10.1111/j.1708-8208.2012.00467.x. PMid:22809239. [ Links ]

22 Yang C, Unursaikhan O, Lee JS, Jung UW, Kim CS, Choi SH. Osteoconductivity and biodegradation of synthetic bone substitutes with different tricalcium phosphate contents in 
rabbits. J Biomed Mater Res B Appl Biomater. 2014 Jan;102(1):80-

8. http://dx.doi.org/10.1002/jbm.b.32984. PMid:23852942. [ Links ]

23 Sawada K, Nakahara K, Haga-Tsujimura M, lizuka T, Fujioka-Kobayashi M, Igarashi K, et al. Comparison of three block bone substitutes for bone regeneration: long-term observation in the beagle dog. Odontology. 2018 Oct;106(4):398-407. http://dx.doi.org/10.1007/s10266018-0352-7. PMid:29557992. [ Links ]

24 Jensen SS, Broggini N, Hjørting-Hansen E, Schenk R, Buser D. Bone healing and graft resorption of autograft, anorganic bovine bone and beta-tricalcium phosphate. A histologic and histomorphometric study in the mandibles of minipigs. Clin Oral Implants Res. 2006 Jun;17(3):237-43. http://dx.doi.org/10.1111/j.1600-0501.2005.01257.x. PMid:16672017. [ Links ]

25 Simunek A, Kopecka D, Somanathan RV, Pilathadka S, Brazda T. Deproteinized bovine bone versus beta-tricalcium phosphate in sinus augmentation surgery: a comparative histologic and histomorphometric study. Int J Oral Maxillofac Implants. 2008 SepOct;23(5):935-42. PMid:19014165. [ Links ]

26 Mordenfeld A, Lindgren C, Hallman M. Sinus floor augmentation using Straumann ${ }^{\oplus}$ BoneCeramic ${ }^{\mathrm{TM}}$ and Bio-Oss ${ }^{\circledR}$ in a split mouth design and later placement of implants: a 5-year report from a longitudinal study. Clin Implant Dent Relat Res. 2016 Oct;18(5):926-36. http://dx.doi.org/10.1111/cid.12374. PMid:26358740. [ Links ]

27 Starch-Jensen T, Aludden H, Hallman M, Dahlin C, Christensen AE, Mordenfeld A. A systematic review and meta-analysis of long-term studies (five or more years) assessing maxillary sinus floor augmentation. Int J Oral Maxillofac Surg. 2018 Jan;47(1):103-

16. http://dx.doi.org/10.1016/j.ijom.2017.05.001. PMid:28545806. [ Links ]

28 Vajgel A, Mardas N, Farias BC, Petrie A, Cimões R, Donos N. A systematic review on the critical size defect model. Clin Oral Implants Res. 2014 Aug;25(8):879-

93. http://dx.doi.org/10.1111/clr.12194. PMid:23742162. [ Links ]

29- Aroni, Mauricio Andrés Tinajero, et al. "Bone repair induced by different bone graft substitutes in critical-sized defects in rat calvaria." Revista de Odontologia da UNESP 48 (2019). 